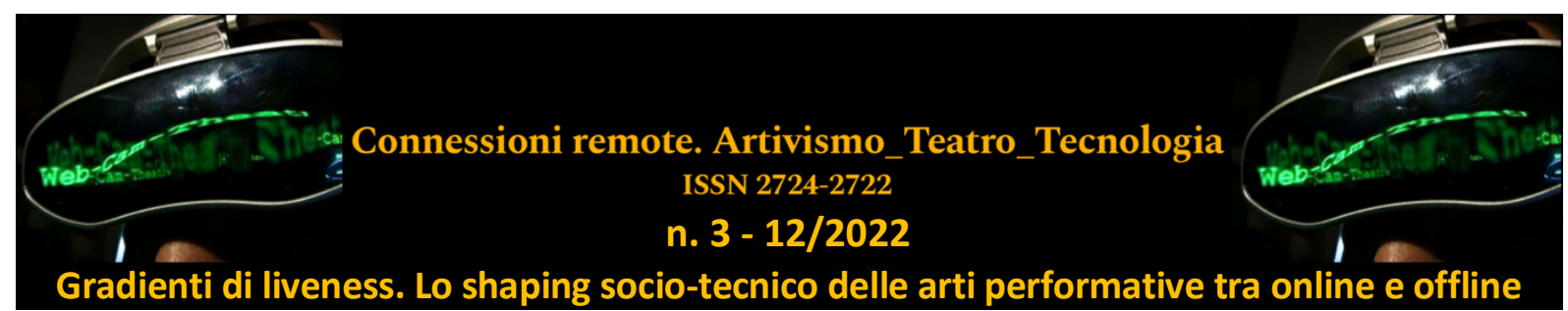

\title{
Bruma/Net e la performance telematica di Kònic Thtr Cover Story: Presentazione della copertina del numero 3
}

Vincenzo Sansone

La pandemia degli ultimi due anni e ancora in corso, provocata dal COVID-19, ha messo in crisi vite e abitudini. Salvo qualche rara eccezione, tutte le attività commerciali e culturali nel primo periodo di lockdown, e in alcuni casi anche oltre, sono state chiuse, tra queste i teatri.

In Italia sono rimasti chiusi quasi in maniera continuativa da marzo 2020 fino ad aprile 2021 (con qualche limitata apertura nei mesi estivi ma con un'affluenza di spettatori contingentata e con regole variabili da decreto a decreto) ma non è andata meglio nel resto del mondo: stagioni sospese, riprogrammate o addirittura rinviate all'anno successivo (sempre con il punto interrogativo sull'andamento della pandemia).

Molti teatri, per ovviare almeno in parte a tale situazione, hanno programmato le stagioni "on-line", spesso spettacoli delle passate stagioni, registrazioni video con una specifica regia per il video, filmati distribuiti in genere in DVD e che adesso diventano "risorse" streaming da vedere "in diretta", intendendo con diretta non la messa in scena in tempo reale dell'evento ma la distribuzione dell'evento registrato on-line per il solo tempo di durata del video, una simulazione delle modalità esplicative dello spettacolo dal vivo, una delle varie e ormai numerose sfumature del concetto di "liveness" diversa da quella classica che è tipica del teatro: «Physical co-presence of performers and audience; temporal simultaneity of production and reception; experience in the moment ${ }^{1}$.

Anche le istituzioni hanno cercato in qualche modo di porre "goffamente" una pezza alla situazione, inventandosi, per esempio, la piattaforma ITsART, la "Netflix della cultura italiana", che per il teatro non rappresenta nulla di nuovo. È la trasposizione on-line di contenuti video inerenti al teatro, quei contenuti che afferiscono all'ampio significato

\footnotetext{
${ }^{1}$ P. Auslander, Liveness: Performance in a Mediatized Culture (2nd ed.). Routledge, New York, 2008, p. 61. Cover Story Connessioni Remote n. 3 - 12/2021
} 
del termine video-teatro, quegli stessi contenuti editati, come già detto, in DVD e che adesso vengono caricati su una piattaforma di streaming on-demand.

Accanto a queste modalità non propriamente innovative, se ne sono sviluppate di altre che, invece, ripensano allo spettacolo dal vivo in prospettiva dell'on-line, non come trasposizione di uno spettacolo dal palcoscenico all'on-line ma come costruzione di una performance dal vivo, che gioca con lo spazio-tempo, riscrivendo i confini della classical liveness. Nulla di nuovo in realtà ma un grande upgrade in termini tecnologici di quanto si è iniziato a sperimentare ormai più di vent'anni fa ma sempre supportato da idee creative. La performance telematica, infatti, ha già una lunga storia e forse alcuni si stanno accorgendo di questa possibilità solo a seguito della costrizione imposta dalla pandemia. Passi da gigante sicuramente sono stati fatti dalle primissime sperimentazioni di performance che impiegano le piattaforme on-line come "palcoscenico" come Waiting for Godot del gruppo artistico Desktop Theater "messo in scena" dentro una chat IRC o gli esperimenti di Web-cam-theatre di Giacomo Verde:

Con l'uso delle web-cam attraverso internet è possibile vedere in tempo reale luoghi e persone lontanissime. Normalmente le web-cam sono usate anche per fare video conferenze. Scopo di questo sito è sperimentare e realizzare dei brevi eventi teatrali attraverso l'uso delle web-cam. Se consideriamo il teatro come la compresenza di attori e spettatori in uno stesso spazio-tempo e se intendiamo il pianeta Terra come un unico spazio-tempo allora possiamo utilizzare il riquadro delle piccole immagini riprese dalle web-cam come se fosse un palcoscenico. Certamente le regole di questo piccolo "palco planetario" non sono le stesse del teatro tradizionale: scopo del progetto Web-CamTheatre è scoprirle e attuarle attraverso la sperimentazione sia in rete che in work-shop ${ }^{2}$.
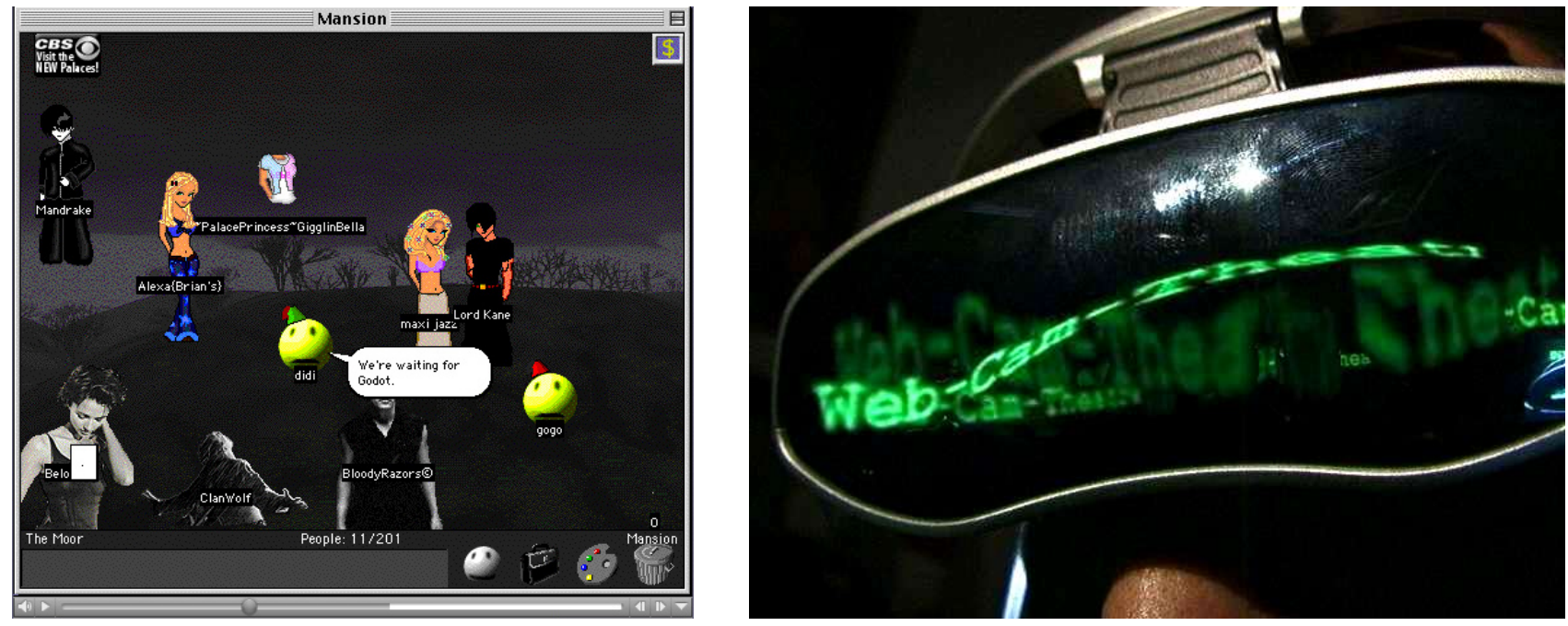

A sinistra Waiting for Godot di Desktop Theater. A destra Web-Cam-Theatre di Giacomo Verde.

${ }^{2}$ G. Verde, descrizione di Web-cam-theatre, maggio 2001, dalla pagina web http://webcamtheatre.org/progetto.htm.

Cover Story

Connessioni Remote n. 3 - 12/2021 
Con lo sviluppo di web-cam sempre più performanti, con la connessione internet sempre più veloce e con la creazione di software di elaborazione digitale e in tempo reale dei segnali audio-video e soprattutto di piattaforme sempre più user-friendly, pronte ad accogliere gli spettatori nella messa in scena su questo immenso palcoscenico che è la Terra, i riquadri delle piccole immagini riprese dalle web-cam sono diventati realmente dei performer che interagiscono in tempo reale e le piattaforme che li accolgono sono adesso dei teatri virtuali con tanto di scenografie interattive.

La copertina del numero 3 di Connessioni Remote è una elaborazione grafica di un'immagine della performance on-line Bruma/Net del gruppo tecno-performativo Kònic Thtr di Barcellona sviluppata a partire dal 2020.

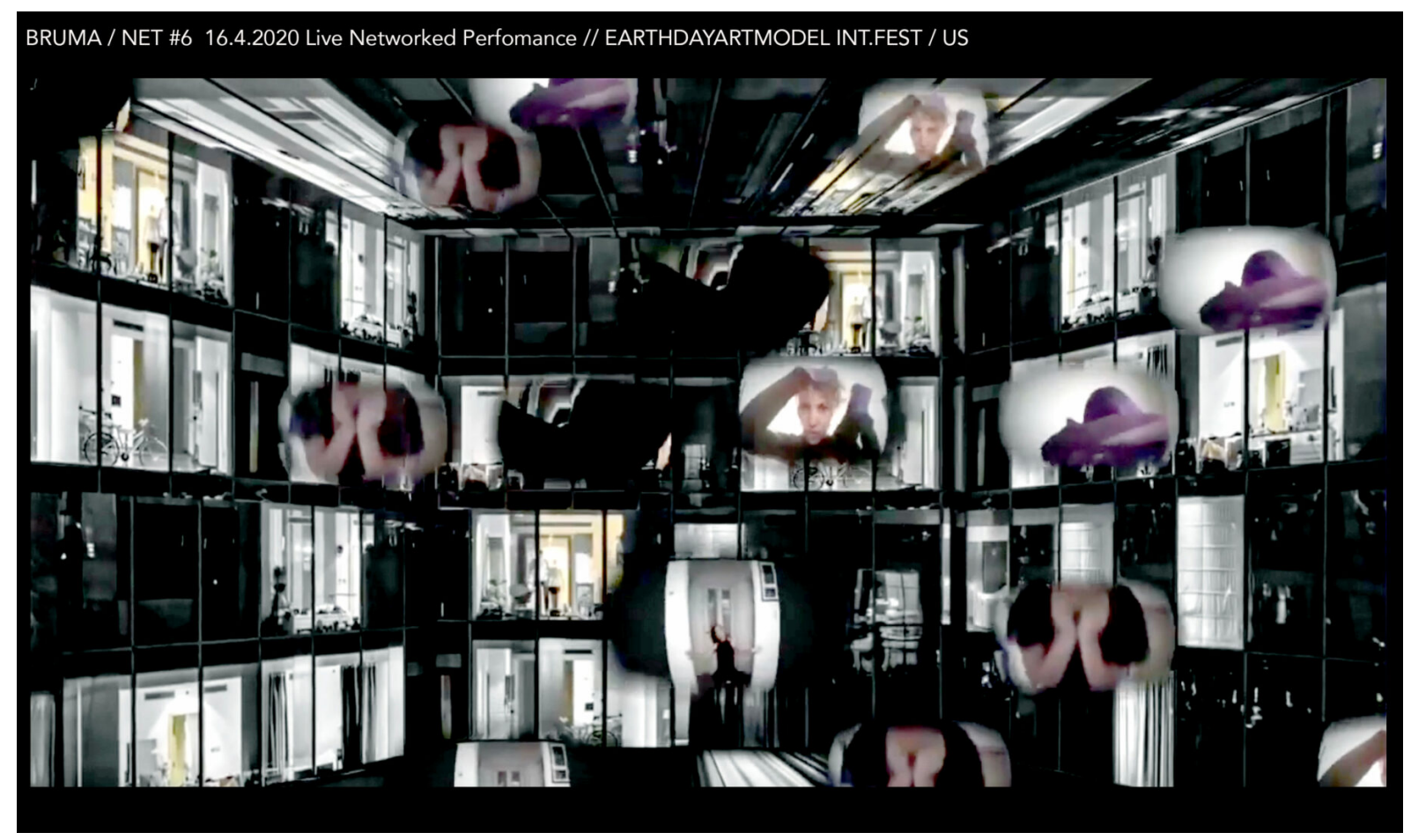

Immagine originale

Le anime creative, concettuali, progettuali ed esecutive di KònicThtr\&Lab sono Rosa Sánchez e Alain Baumann che iniziano il lavoro sperimentale di connessione tra arti performative e tecnologie già all'inizio degli anni Novanta:

Rosa: Ho incontrato Alain nel 1990, anche se in realtà Kònic è nato nel 1985 e ancor prima di fondare i Kònic ero interessata a ciò che in quel periodo era chiamato teatro visivo. Teatro visivo significava integrazione di differenti linguaggi, di differenti discipline che lavoravano insieme per raggiungere un'unità globale. Ho studiato presso I'Istituto del Teatro, occupandomi 
anche di marionette. Iniziai perché avevo bisogno in quel momento di ottenere le risposte per le numerose domande che stavo chiedendo a me stessa come, per esempio, che cosa volessi fare o che tipo di espressione teatrale e performativa avessi voluto intraprendere. In altre parole, stavo ricercando quale tipo di linguaggio e quale tipo di espressione erano le migliori in relazione al mio interesse per la comunicazione. Come ho detto ho studiato anche teatro di marionette e ho lavorato anche con manichini che avevano le mie stesse dimensioni. Quando ho fondato i Kònic ho avuto la possibilità di realizzare produzioni con molte persone sempre su questa idea di teatro visivo, fin quando non incontrai Alain. lo già lavoravo con dispositivi ottici e analogici e insieme abbiamo perseguito questa ricerca teatrale che fa uso anche di tecnologie, sempre tenendo in mente le questioni fondamentali che hanno basato l'inizio del mio percorso, ma elaborando al tempo stesso nuove questioni cui tentar di dare una risposta e producendo spettacoli con l'idea del teatro visivo ma inserendo anche le nuove tecnologie informatiche apportate da Alain e cominciando anche a studiare concetti come virtualità e alterità.

Alain: L'interattività è la ragione principale del perché lavoriamo nel campo delle arti performative, l'improvvisazione è parte della maniera con la quale ci esprimiamo e l'interattività è un modo per modificare la risposta dei paesaggi. Lavorare a progetti interattivi è sempre un lungo processo di studio che dipende dal cosa vuoi dire ma allo stesso tempo individuare il miglior strumento per poterlo dire. Per esempio, se vogliamo parlare del controllo utilizziamo videocamere di controllo, videocamere di sorveglianza chiedendoci cosa possiamo fare con esse. Dai primi anni Novanta abbiamo lavorato con diversi tipi di tecnologia, anche se all'inizio fu molto difficile poterci lavorare. Molte persone pensavano che l'interattività fosse qualcuno con un computer ma in realtà non è solo questo. È sempre una tua performance con il pubblico mediata dalla nuova interattività permessa dal computer per cui l'oggetto principale è sempre l'interagire con le persone ${ }^{3}$.

I progetti di Kònic Thtr impiegano vari media, tra cui la rete internet e dispositivi mobili connessi a essa durante le performance, suddividendosi spesso in escena distribuida (performance telematica) ed escena interactiva (performance interattiva), due modalità che nella maggior parte dei casi si incontrano.

L'interesse per la performance telematica, dunque, nasce molto tempo prima del lockdown, della pandemia e della "costrizione" del dover utilizzare la rete per interagire con gli altri e per fare arte. La sperimentazione di Kònic con la performance telematica è sempre stata basata sull'idea di connettere diversi spazio-tempo locali, diversi nodi, all'interno dello spazio-tempo globale che è la Terra, sperimentando in maniera del tutto

\footnotetext{
${ }^{3}$ Parole tratte dall'intervista personale a Kònic Thtr a cura di V. Sansone realizzata per il progetto di ricerca dottorale. Per un'analisi di alcuni aspetti delle attività performative e di alcune performance di Kònic Thtr cfr. V. Sansone, Scenografia digitale e interattività. II video projection mapping nuova macchina teatrale della visione, Aracne, Roma 2021. Sulla performance telematica di Kònic Thtr cfr. anche V. Sansone, Le arti performative nel mondo di Internet e dell'Interaction Design: I'avventura tecno-artistica di Kònic Thtr di Barcellona, in Conferenza GARR 2016 - The Creactive Network. Uno spazio per condividere e creare nuova conoscenza - Selected Papers - Firenze, 30 novembre-2 dicembre 2016, pp. 118-122, Associazione Consortium GARR, Roma 2017, https://www.eventi.garr.it/it/conf16/home/materiali-conferenza-2016/selected-papers/155-conf16-selectedpapers-21-sansone.
} 
differente il principio che sottende le arti performative, la presenza, in un nuovo paradigma, quello della presenza in assenza, quell'assenza spaziale locale che però c'è sempre a livello globale e rafforzata da un tempo che, al di là dei diversi fusi orari, è lo stesso nella condivisione. Le performance telematiche di Kònic sono performance in cui la drammaturgia diventa visiva: è l'elemento audio-visivo che permette a due danzatori dislocati in due parti differenti del mondo di danzare assieme su un unico palcoscenico virtuale.

A fungere da base della drammaturgia sono i corpi dei performer che danzano, che diventano immagini in video che, a loro volta, vengono coreografate digitalmente e immesse on-line per raggiungere i diversi nodi locali. Lo spettatore di queste performance è in generale uno spettatore che assiste solo in parte alla performance secondo i dettami della classical liveness. Dal vivo, infatti, vede solo la parte che si svolge nel nodo fisico in cui si trova. Per il resto, deve fondere il flusso di immagini che riceve dalla rete con quello che vede in presenza. La performance, dunque, in ogni luogo-nodo risulterà unica, anche se il flusso di immagini ricevuto sarà diverso. Il passaggio tra spazio fisico e virtuale diventa senza soluzione di continuità e fa sì che l'immagine proveniente dal nodo distante diventi un corpo che è anche "presente", "dal vivo".

Questa sperimentazione è affiancata da Kònic a una sperimentazione che, invece, avviene esclusivamente on-line, dove i diversi nodi locali interagiscono e si incontrano tra loro e per gli spettatori solo nella dimensione virtuale della rete. Ogni performer esegue il suo pezzo nel suo spazio e Kònic costruisce uno spazio unico e virtuale dentro cui le varie parti vengono immesse, creando una coreografia virtuale.

E si ritorna al lockdown, ai teatri e agli spazi culturali chiusi. Unica modalità di sperimentazione artistica non può che essere la dimensione dell'on-line. Kònic, dunque, si trova preparata per questa situazione, non improvvisa, non mette in streaming materiali d'archivio ma crea performance esclusivamente per la rete, in cui l'ultima modalità di performance telematica descritta, quella della coreografia virtuale, trova ampia diffusione. Nasce così nel 2020, proprio a inizio lockdown, il progetto Brauma/Net:

La Pandemia, el aislamiento derivado de ella, ha hecho que muchos artistas escénicos recurrieran a las prácticas performáticas online, convirtiendo estos formatos en las producciones más utilizadas en esta fase de cuarenta mundial. Los formatos de danza para la cámara y videodanza, ya conocidos por los jóvenes coreógrafos, toman ahora un 
nuevo significado y dan el salto conceptual de ser clasificadas en el ámbito de lo Digital. Bruma/Net es un proyecto que la Compañía de danza Intermedia Kònic Thtr ha diseñado y producido desde su casa en Barcelona, durante el Confinamiento causado por la Emergencia sanitaria del COVID19. En los meses de obligado aislamiento, los directores de esta compañía han tomado iniciativas solidarias para ayudar a que los artistas de todo el mundo pudieran seguir artísticamente activos y contribuir a aliviar su soledad ${ }^{4}$.

Si tratta di una performance dal vivo che viene messa in scena in rete, una performance che ibrida diversi linguaggi come danza, musica, fotografia, video e letteratura. Ogni partecipante-performer collegato dal proprio spazio di residenza o di isolamento, offre la sua performance che viene composta dal vivo da Kònic Thtr e trasposta in una esperienza di video-arte-teatro per lo spazio digitale.

L'immagine scelta per la copertina esplica alla perfezione questa idea di singole performance che trovano un'unità nello spazio virtuale. Ciascuna finestrella delle varie web-cam è incasellata dentro un unico frame audio-visivo che arriva nella sua totalità nelle case di chi osserva. Nello specifico, per la copertina di Connessioni Remote 3 è stato applicato uno stretch verticale all'immagine originale con un semplice intento: voler restituire quasi la controparte di quel che si vede.

Se l'immagine che osserviamo è quella della performance inquadrata dal punto di vista dello spettatore, la cornice del suo computer, lo stretch vuole suggerire l'ipotetico specchiamento di quella immagine, suggerire l'idea di quegli spettatori che osservano quella performance dal vivo ma da casa e su internet, suggerire un'analogia visiva con lo spazio teatrale per eccellenza, il teatro all'italiana. Ecco che quell'immagine diventa un insieme di palchetti teatrali, quei palchetti che nella loro origine erano delle case private. Si chiudeva la tenda e il proprietario poteva fare altro dal vedere lo spettacolo.

In qualche modo è quello che succede nella fruizione on-line del teatro: le nostre case sono diventate tanti palchetti da cui assistere a un evento ma da cui ci si può anche assentare per qualche secondo, perdendosi però qualcosa, non potendo premere il tasto rewind per tornare indietro, perché la performance si sta svolgendo on-line, qui e ora.

Di seguito si riporta un'immagine che mostra i palchi del Teatro alla Scala di Milano e sotto l'immagine della copertina di Connessioni Remote 3.

\footnotetext{
${ }^{4}$ Kònic Thtr - Descrizione del progetto Bruma/Net
} 

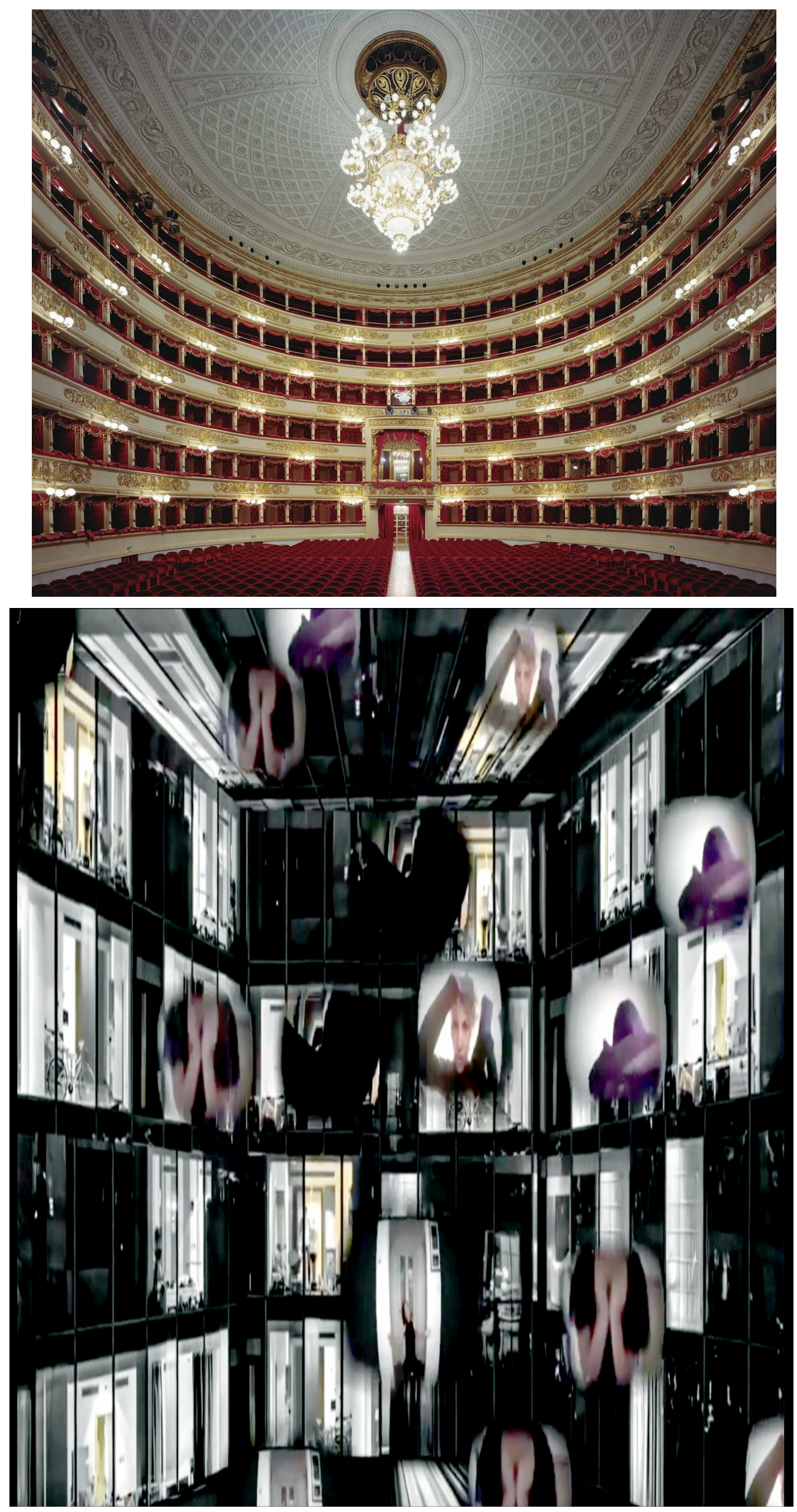
Alcune immagini dei vari interventi di Bruma/Net
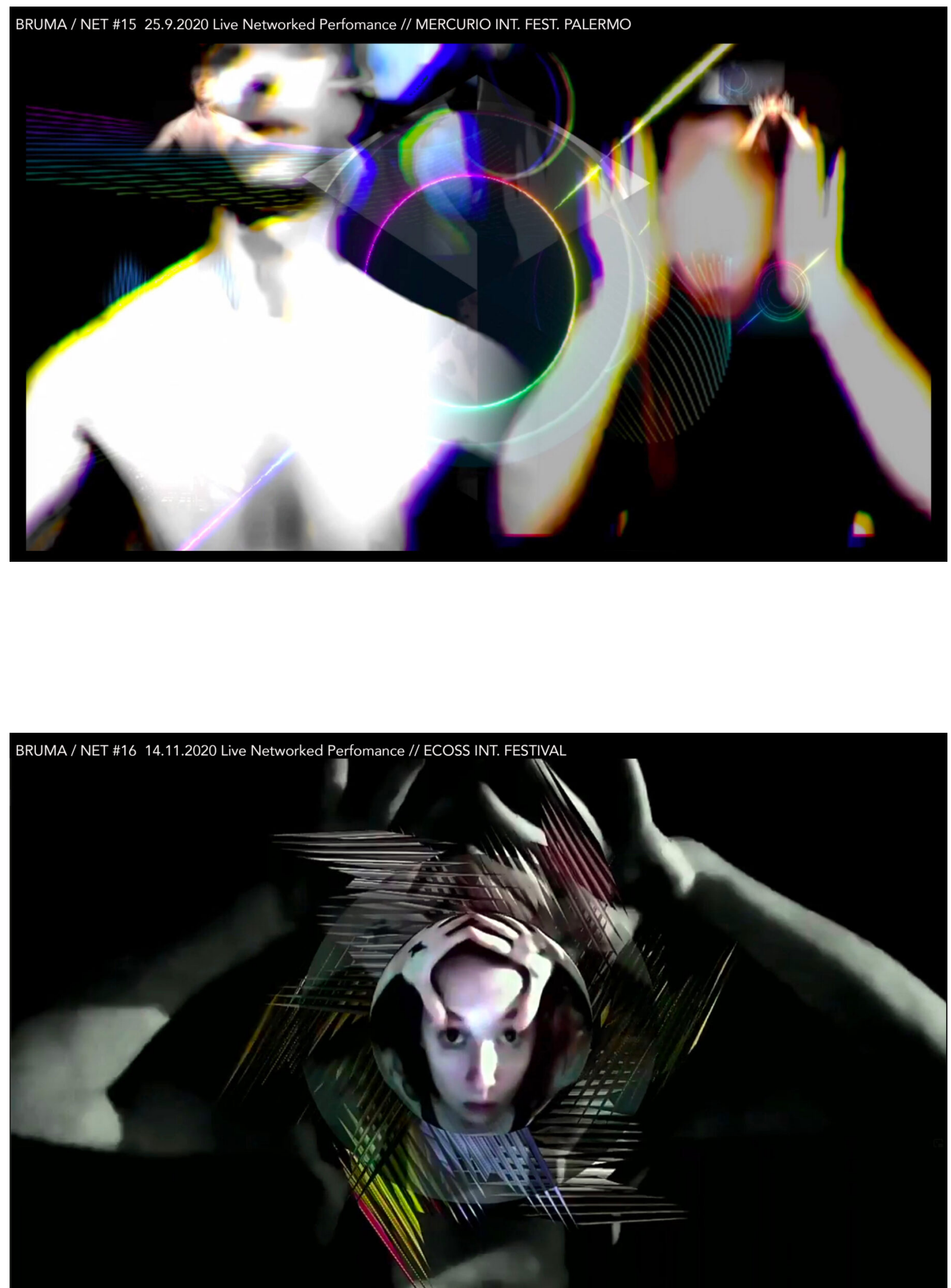

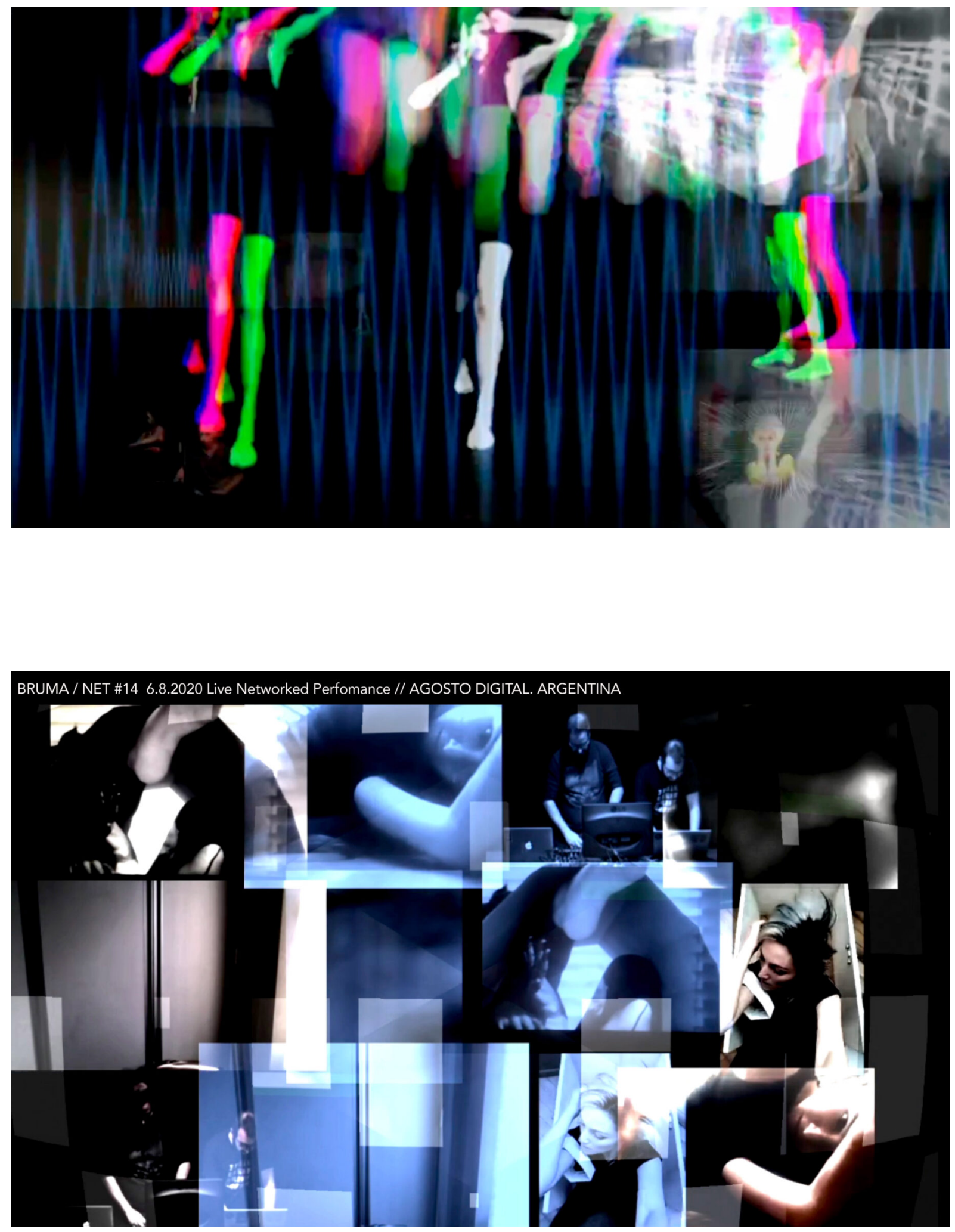